ROCZNIKI TEOLOGICZNE

Tom LXVIII, zeszyt $2-2021$

DOI: https://doi.org/10.18290/rt21682-3

ANTONIO PANARO

\title{
GIOVANNI DUNS SCOTO: UN TEOLOGO INCOMPRESO, UN SANTO DA RISCOPRIRE
}

\author{
JOHN DUNS SCOTUS: \\ A MISUNDERSTOOD THEOLOGIAN, A SAINT TO BE REDISCOVERED
}

\begin{abstract}
A bstract. In approaching the thought of John Duns Scotus, one inevitably encounters the luminous life testimony of a great saint. One is dealing with "an inseparable whole of philosophy, theology and spirituality." John Duns Scotus, 13th century philosopher and theologian, is a controversial and original author. His works in the past have had more critics and opponents than supporters and disciples. He is a more modern thinker than one might imagine. He is by no means one of the many theologians and philosophers of the past, nor are his writings just gathering dust in our libraries. "He is not a fossil to be admired, but a living and throbbing Master, full of ardour and valuable insights" of great relevance. This Scottish-born Franciscan, born between 1265/66 and died in Cologne on the 8th November 1308, far from his homeland, is a theologian and a saint of great depth that we can perhaps only begin to appreciate today.
\end{abstract}

Keywords: theology; holiness; doctrine; reason; faith.

\section{UN BREVE PROFILO TEOLOGICO E BIOGRAFICO}

Nell'approccio al pensiero di Giovanni Duns Scoto, ci si imbatte in maniera inevitabile con la luminosa testimonianza di vita di un grande santo. Si ha a che fare con ,un insieme inseparabile di filosofia, teologia e spiritualità. [...] Scoto ha una visione personalista e unitaria del pensiero e dell'azione, unità che riceve

Dott. Antonio PANARo - presbitero dell'Arcidiocesi di Varsavia, formatore nel Seminario Arcidiocesano Missionario Redemptoris Mater, docente presso l'Accademia Cattolica di Varsavia; indirizzo di posta: Akademia Katolicka, ul. Dewajtis 3, 01-815 Warszawa; e-mail: apanaro78@gmail.com; ORCID: https://orcid.org/0000-0003-0048-9056. 
dalla ragione illuminata dalla fede e non dalla ragione pura [...] di stile kantiano". Non discrimina la ragione rispetto alla fede, bensì analizza l'esperienza della ragione separatamente dalla fede e in essa vi trova "luce e coerenza"2.

Giovanni Duns Scoto, filosofo e teologo del XIII secolo è un autore controverso e originale. Le sue opere in passato hanno avuto più critici e oppositori che sostenitori e discepoli ${ }^{3}$. È un pensatore più moderno di quanto ci si possa immaginare, tanto che gli è stato attribuito proprio l'appellativo di modernus $^{4}$.

Giovanni Duns Scoto non è per niente uno fra $\mathrm{i}$ tanti teologi e filosofi del passato, né i suoi scritti servono solo ad impolverare le nostre biblioteche. „Non è un fossile da ammirare, ma è un Maestro vivo e palpitante, pieno di ardore e di preziose intuizioni" di grande attualità ed ispirazione, per approfondire ancora oggi „realtà dottrinali e vitali della Chiesa e del mondo" 5 .

Questo francescano di origine scozzese, nato trail 1265/66 e morto a Colonia l'8 novembre del 1308 lontano dalla sua patria, è un teologo ed un santo di grande spessore che solo oggi forse possiamo iniziare ad apprezzare.

Per quanto l'epitaffio sulla sua tomba non abbia bisogno di commenti riguardo alla durezza del suo definitivo esilio, accettato da lui con fede e temperanza: "Scotia me genuit, Anglia me suscepit, Gallia me docuti, Colonia me tenet".

La sua vita intellettuale è alquanto dinamica, ricca e purtroppo spesso in salita per le difficoltà procurategli nel conseguimento dei titoli accademici. Lo si può notare, elencando il numero dei suoi viaggi, le prestigiose università che frequenta e presso le quali si forma, i personaggi che incontra. Cose queste che contribuiscono efficacemente alla sua istruzione filosofica e teologica ${ }^{6}$.

\footnotetext{
${ }^{1}$ José A. Merino, Storia della Filosofia Francescana (Milano: Edizioni Biblioteca Francescana, 1993), 227-8.

${ }^{2}$ Vedi Merino, Storia della Filosofia, 228.

${ }^{3}$ Cfr. Romuald H. Kośla, Jan Duns Szkot. Jego dzieto i myśl od początku XX wieku do dzisiaj (Kraków: Wydawnictwo Unum, 2011), 5-6.

${ }^{4}$ Cfr. Kośla, Jan Duns Szkot, 245-88.

5 Vedi Barnaba Hechich, „L'edizione critica delle opere del Beato Giovanni Duns Scoto. Traguardi e prospettive”, in Błogosławiony Jan Duns Szkot 1308-2008. Materiaty Międzynarodowego Sympozjum Jubileuszowego z okazji 700-lecia śmierci bt. Jana Dunsa Szkota, Katolicki Uniwersytet Lubelski Jana Pawła II, 8-10 kwietnia 2008, a cura di Edward I. Zieliński OFMConv e Roman Maieran (Lublin: Wydawnictwo KUL, 2010), 74.

${ }^{6}$ Cfr. Francesco Fiorentino, Il Prologo dell'Ordinatio di Giovanni Duns Scoto. Introduzione, testo, traduzione e commento (Roma: Città Nuova Editrice, 2016), 15-7. Cfr. Guido Alliney, Giovanni Duns Scoto. Introduzione al pensiero filosofico (Bari: Edizioni di Pagina, 2012), 5-7. Vedi
} 
Rileggere la biografia di Scoto unita alla presentazione della sua dottrina, è questa, a nostro avviso, la scelta metodologica migliore. "Il pensiero di Scoto è l'essenzializzazione di una profonda esperienza di vita cristiana"7. Per quanto quest'ultima non traspaia esplicitamente dai suoi scritti, tuttavia la santità di vita, l'amore per Cristo e per la novità del Vangelo che diffonde da teologo, sono queste che spingono il Sottile al confronto razionale con la filosofia pagana.

Scoto preferisce mostrare un netto distacco tra la struttura essenziale dei trattati e la sua vita spirituale. Forse il motivo di questa scelta è quello di procedere con rigore scientifico nella sua analisi per custodire per quanto possibile la massima obiettività nel dialogo tra ragione e fede.

Nella rilettura del taglio profondamente teoretico del pensiero di Scoto non ci si deve dimenticare del profilo esistenziale del suo discorso, che "ne costituisce il nerbo e lo sfondo permanente" ${ }^{\text {. }}$. Bisogna infatti ricordare che tutti gli autori medioevali "avevano sempre presente ed operante nella loro spiritualità tutta la speculazione pre-scolastica, patristica e agostiniana, monastica e bernardina" 9 .

Dopo aver studiato filosofia come novizio dei frati minori in Scozia, si trasferisce a Northampton dove completa gli studi di teologia e viene ordinato sacerdote il 17 marzo 1291 dal vescovo di Lincoln. Prima di trasferirsi a Parigi, soggiorna ancora ad Oxford per tre anni (1291-1293) per proseguire gli studi teologici sotto il magistero di Guglielmo di Ware. A Parigi invece tra il 1293-1295 si trasferisce per seguire le lezioni di Gonsalvo di Spagna e conseguire il dottorato in teologia nel 1297. Qui ha già iniziato ad insegnare in veste di baccelliere biblico e ,sententiarius" ${ }^{\prime 10}$. Il dottorato non gli viene

anche per una bibliografia essenziale: Charles K. Brampton, "Duns Scotus at Oxford, 1288-1301", Franciscan Studies 24 (1964), 5-20; Roberto Zavalloni, Giovanni Duns Scoto. Maestro di vita e pensiero (Bologna: Edizioni Francescane, 1992); William J. Courtenay, Scotus at Paris, in Via Scoti. Methodologia ad mentem Joannis Duns Scoti. Atti del Congresso Scotistico Internazionale, Roma 9-11 marzo 1993, a cura di Leonardo Sileo, vol. 1 (Roma: Edizioni Antonianum, 1995), 149-63; Allan B. Wolter, Duns Scotus at Oxford, in Via Scoti, 183-92; Olivier Boulnois, La rigueur de la charité (Paris: Les Éditions du Cerf, 1998); Antonie Vos, The philosophy of John Duns Scotus (Edinburgh: Edinburgh University Press, 2007).

${ }^{7}$ Orlando Todisco, „La ragione nella fede secondo G. Duns Scoto”, Miscellanea Francescana 77 (1977): 263.

8 Todisco, ,La ragione”.

${ }^{9}$ Armando Rigobello, Linee per una antropologia prescolastica (Padova: Edizioni Antenore, 1972), 39.

${ }^{10}$ Cfr. Alessandro Ghisalberti, Giovanni Duns Scoto: filosofia e teologia (Milano: Edizioni Biblioteca Francescana, 1995), 99. 
concesso e Scoto sembra essersi spostato a Cambridge nel 1297-1300 (come dice egli stesso nel primo libro dell'Ordinatio, prima ancora di tenere lezione ad Oxford, 1300-1301) ${ }^{11}$. Durante questo suo secondo soggiorno ad Oxford commenta i primi tre libri delle Sentenze di Pietro Lombardo ${ }^{12}$. A Oxford partecipa al dibattito presieduto dal maestro Filippo Bridglinton e nel giugno del 1302 viene richiamato a Parigi, dove ripete la letture dei primi tre libri delle Sententiae per tutto l'anno accademico successivo. Ivi prende parte al dibattito tra Gonsalvo di Spagna e Giovanni Eckhart sulla visio beatifica. Le fonti dicono che il suo assistente di allora era Tommaso di Dunstan, che poi sarà designato maestro in carica ad Oxford nel 1320 . L'essersi schierato dalla parte di Papa Bonifacio VIII nel suo conflitto ideologico-politico contro il re di Francia Filippo il Bello, purtroppo gli costò caro. Dovette abbandonare Parigi nel 1303 per trovare rifugio nuovamente ad Oxford, dove commenta il III libro delle Sententiae (1303-1304). La morte del papa e l'elezione di Benedetto XI riportano pace e serenità nel clima politico francese. Scoto insieme ad altri francescani esiliati ritorna a Parigi per completare la lettura del IV libro delle Sententiae. In questo periodo stando alle fonti è registrata una disputa nella quale assume il ruolo in aula di respondens. La questione riguardava la fruizione beatifica e tra i partecipanti si menziona il baccelliere francescano Egidio di Longny, Goffredo di Fontaines ed un certo 'Alano' (un maestro francescano di dubbia identità) ${ }^{13}$.

Giovanni Duns Scoto conseguirà solo quattro anni prima della sua morte il tanto aspirato e meritato titolo di Magister ${ }^{14}$ : era il 18 novembre del 1304. Gonsalvo di Spagna divenuto ormai Ministro Generale dell'Ordine dei Minori promosse la carriera accademica di Scoto, accelerandone i tempi con una bella

\footnotetext{
${ }^{11}$ Cfr. Fiorentino, Il Prologo dell'Ordinatio, 15.

${ }^{12}$ Le Sententiae di Pietro Lombardo rappresentavano il principale manuale di teologia dogmatica dell'epoca, che l'aspirante al titolo di maestro doveva commentare. Il repertorio tematico era vastissimo: logica, semantica, gnoseologia, epistemologia, etica, politica, economia, ontologia e metafisica.

${ }^{13}$ La disputa è registrata in Reportata Parisiensia III, d. 18, q. 3, a. 2; cfr. Giovanni Duns Scoto, „Collectationes Oxonienses et Parisienses”, in Opera omnia, a cura di Giovanni Lauriola, II/1 (Bari: AGA Alberobello, 1999), 1092-7. Altri come William J. Courtenay sono del parere che questa disputa corrisponda all'inceptio di Egidio di Longny, cfr. „Early Scotist at Paris”, Franciscan Studies 69 (2011): 195-6.

${ }^{14}$ Il titolo di Magister, cioè di Maestro della Santa teologia, rappresentava a quei tempi, nel Medioevo, il massimo riconoscimento, che rendeva idonei alla direzione di una scuola a statuto proprio ed indipendente - vedi Mieczysław A. Krąpiec, „Przesłanie”, in Błogosławiony Jan Duns Szkot 1308-2008, 20.
} 
lettera in suo sostegno. La disputa con il maestro domenicano Guglielmo di Pietro di Godino nel 1305 inaugura il magistero di Scoto e contemporaneamente al successo ne segna il suo martirio intellettuale e spirituale nel 1307 . La disputa riguarderà il tema del principio d'individuazione, l'Immacolata Concezione ed alcune questioni quodlibetali (Avvento del 1306 - Quaresima del 1307). Verso la fine del 1307 su decisione di Gonsalvo viene inviato ufficialmente a Colonia come lettore. È in realtà un atto di generosità del Generale dell'Ordine in difesa di Scoto, che nonostante tutto lascerà nel cuore del santo una profonda ferita per aver subito l'ennesimo esilio, sopportato in silenzio e con obbedienza alla volontà di Dio. Muore all'età di 43 anni l'8 novembre del 1308 a Colonia.

\section{LA RISCOPERTA DI UN SANTO}

Solo oggi il nome ed il pensiero di Giovanni Duns Scoto cominciano ad emergere dall'oscurità del dimenticatoio del passato. Siamo infatti testimoni dei suoi primi bagliori di luce e di risurrezione.

Di nessun autore francescano esistono tanti manoscritti, nelle varie biblioteche del mondo, quanti ce ne sono di Duns Scoto. Il suo insegnamento, già ripreso dai discepoli nel corso delle lezioni in aula, era ricercato e richiesto dappertutto, non solo nell'Ordine, ma in tutti gli ambienti di studio e di apostolato. [...] Dopo la sua morte, Duns Scoto è presente in ogni autore: non lo si può ignorare! ${ }^{15}$

Ci sono voluti secoli di rifiuti, attacchi, minaccie, calunnie e falsità sul suo conto, che hanno infangato la sua fama purtroppo fino ad oggi ${ }^{16}$. Duns Scoto è stato persino accusato di eresia.

Dopo quattro, cinque secoli la dottrina di Duns Scoto è tornata ad essere insegnata nelle università del mondo. Lo stesso Karol Wojtyła né stato uno dei propagatori durante i suoi anni di insegnamento all'Università Cattolica di Lublino (che oggi porta il Suo nome - Katolicki Uniwersytet Lubelski Jana Pawła II ${ }^{17}$. Non ci si può infatti dimenticare che il prof. Wojtyła assieme ad un folto gruppo di colleghi ${ }^{18}$ ha promosso presso la stessa università

\footnotetext{
${ }^{15}$ Hechich, „L'edizione critica delle opere”, 73.

${ }^{16}$ Cfr. Hechich, „L'edizione critica delle opere”, 67-70.

${ }^{17}$ Cfr. Krąpiec, „Przesłanie”, 21.

${ }^{18}$ In quel gruppo vi erano: Mieczysław A. Krąpiec, Wincenty Granat, Karol Wojtyła, Marian Kurdziałek, Edward I. Zieliński.
} 
varie dissertazioni dottorali e pubblicazioni di vario tipo, cosa che conferma il riconoscimento accademico dell'indiscussa autorità di Scoto ai nostri giorni ${ }^{19}$.

La verità viene prima o poi a galla e così è stato anche per Duns Scoto. La Verità che è Cristo ( $\mathrm{cfr}$. Gv 14,6) non è data una volta per tutte, né la si raggiungerà mai con le sole forze umane. Eppure Cristo ci ha assicurato che lo Spirito Santo ci insegnerà (cfr. Gv 14,26$)$ e ci guiderà alla verità tutt'intera (cfr. Gv 16, 13). È proprio ciò di cui era convinto Duns Scoto, che continuando il pensiero di S. Gregorio Magno, afferma senza esitazione: „In processo generationis umane semper crevit notitia veritatis” - „nel cammino del genere umano, la conoscenza della verità è sempre in crescita" ${ }^{20}$.

Esiliato ed emarginato sia dal mondo accademico per il suo pensiero troppo innovativo e rivoluzionario rispetto ad i canoni imposti, sia da una Chiesa che ancora non aveva compreso né il suo spessore intellettuale né la sua santità. Egli è stato un figlio incompreso che però ha sempre amato la Madre Chiesa ed in spirito di obbedienza Le è stato sempre sottomesso.

Le accuse contro di lui e contro la sua dottrina, formulate nei secoli passati, in realtà si possono raggruppare in due specie e al tempo stesso ne spiegano le motivazioni: 1 . erano dovute ,alla falsa trasmissione dei testi, opera di inesperti amanuensi, e alle edizioni imperfette"; 2 . dovute a partigianeria per una scuola in lotta contro le altre" ${ }^{21}$. Scoto appartiene alla schiera di quei geni incompresi che per onestà intellettuale non possono omologarsi al pensiero delle masse, perciò la sua originalità e sottigliezza di analisi è stata anche la causa principale delle sue sofferenze. Le sue opinioni prendevano le distanze dai grandi maestri che lo avevano preceduto e soprattutto dalla Scolastica (Alberto il Grande, Tommaso d'Aquino, Alessandro di Harles, Bonaventura da Bagnoregio) $)^{22}$. La dottrina di Tommaso d'Aquino era stata così assimilata dal Magistero ecclesiale, fino al punto che tutto ciò che era contrario a Tommaso veniva ritenuto automaticamente contrario alla Chiesa e perfino eretico ${ }^{23}$.

Bisogna comprendere il contesto storico-filosofico medioevale in cui la dottrina e gli scritti del nostro Dottore Sottile si collocano, per renderci conto

\footnotetext{
${ }^{19}$ Cfr. Stanisław Wilk, „Siedemsetlecie śmierci bł. Jana Dunsa Szkota”, in Błogosławiony Jan Duns Szkot 1308-2008, 14.

${ }^{20}$ Duns Scotus, Ordinatio IV, d. 1., n. 256 (ed. Vat. XI)

${ }^{21}$ Hechich, „L'edizione critica delle opere”, 67.

${ }^{22}$ Cfr. ibidem, 67; cfr. anche Kośla, Jan Duns Szkot, 5.

${ }^{23}$ Cfr. Hechich, ,L'edizione critica delle opere”, 67.
} 
della statura morale e del genio intellettuale dello stesso. "Gli studiosi di Duns Scoto fanno rilevare che il Dottore francescano si trovava, dopo la morte di Tommaso d'Aquino $(\dagger 1274)$, di fronte alle recenti condanne parigine (1277) di certe dottrine e di certe tesi sostenute dagli ambienti filosofici (della "Facoltà delle arti") e già da tempo contrastate dal suo predecessore Bonaventura e, in genere dall'indirizzo filosofico-teologico che si rifaceva alla prospettivo platonico-agostianiana e non certo a quella aristotelico averroistica" 24 .

Ponendoci la domanda circa la genesi storica del pensiero scotista dobbiamo rifarci all'opinione di illustri medievalisti come F. Van Steenberghen (†1993) che parla della seconda metà del XIII secolo, periodo in cui il giovane Giovanni Duns Scoto fece i suoi primi passi in filosofia e poi in teologia, come quel "breve" e "forse il più attraente del Medioevo per la storia del pensiero" 25 . Un terzo di secolo separa la morte del Dottore Sottile $(\dagger 1308)$ da quella dei suoi grandi predecessori cristiani: Tommaso d'Aquino e Bonaventura (†1274). Il fascino di quell'epoca era dettato dal tentativo degli studiosi di rispondere ad uno sconcertante enigma: come sarà mai stato possibile passare dalle grandi sintesi del Dottore Serafico da una parte e dell'Angelico dall'altra per giungere ad una profondamente diversa dai primi, quella del Sottile? Van Steenberghen risponde che Scoto assimila in sé tutti gli elementi di quell'epoca la predominante priorità del pensiero aristotelico nei circoli filosofici, gli influssi contrastanti neoplatonici ed agostiniani, il clima di ostile antagonismo creatosi tra filosofi e teologi, tra la facoltà delle arti e quella di teologia, a seguito delle condanne di Parigi e di Oxford del marzo del 1277. Tutto ciò "accentuò la sfiducia dei teologi di fronte alla filosofia" ${ }^{26}$.

${ }^{24}$ Angelo Marchesi, "Filosofia e teologia in Giovanni Duns Scoto", in Giovanni Duns Scoto: filosofia e teologia, a cura di Alessandro Ghisalberti (Milano: Edizioni Biblioteca Francescana, 1995), 14.

${ }^{25}$ Fernand van Steenberghen, „La philosophie à la veille de l'entrée en scène de Jean Duns Scot", in Atti del Congresso scotistico internazionale. De doctrine J. Duns Scoti, a cura di Leonardo Sileo, vol. 1 (Roma: Edizioni Antonianum, 1968), 65.

${ }^{26}$ Van Steenberghen, „La philosophie”, 72. 


\section{L’EDIZIONE CRITICA DELLE OPERE}

La pubblicazione dell'edizione critica di tutte le opere di Scoto da parte della Commissione Scotista Vaticana ${ }^{27}$ ha fatto cadere la maggior parte delle obiezioni sostenute e ne ha dimostrato l'infondatezza: „Si trattava di obiezioni prese da opere non autentiche di Scoto, ma falsamente a lui attribuite" 28 .

Il 21 aprile del 1938 segna invece la data ufficiale dell'istituzione della prima Commissione Scotista Vaticana con sede a Roma, nei pressi dell'Antonianum ${ }^{29}$. L'incarico di presiedere la stessa fu affidato dal Governo dell'Ordine a P. Carlo Balić (famoso teologo e mariologo di origine croata). „Il materiale fotografico dei manoscritti accumulato a Quaracchi fu trasferito a Roma, altro materiale fotografico e microfilmato fu ordinato e raccolto dalla Commissione, oltre ad una gran quantità di dati storici integrativi, necessari a realizzare una edizione critica seria, degna di questo nome" ${ }^{30}$. Gli eventi bellici della Seconda Guerra Mondiale intralciarono i progetti della Commissione di pubblicare nel più breve tempo possibile i primi volumi. Ciò avvenne nel 1950 quando vennero stampati i primi due volumi dell'Opera omnia. Fino ad oggi la Commissione si vanta della pubblicazione di ben 21 volumi dell'Opera Omnia di Scoto. „I primi quattordici (vol. I-XIV) attengono all'Ordinatio, il principale commento del Doctor Subtilis ai quattro libri delle Sentenze di Pietro Lombardo, personalmente rivisto dal Maestro per la pubblicazione. I volumi dal XVI al XXI, contengono, invece, la Lectura, cioè il testo delle lezioni che Scoto andava preparando per i suoi corsi e che costuiscono la base della posteriore Ordinatio. Il volume XV, diviso in due tomi, di cui il primo è già disponibile (vol. XV.1), contiene gli indici delle due opere" ${ }^{\prime 31}$.

${ }^{27}$ Il Capitolo Generale dell'Ordine dei Frati Minori che si tenne ad Assisi nel 1927 decise di fare fronte alla fioritura degli studi e alla crescita dell'interesse per i grandi maestri teologi del Medioevo, come anche per Duns Scoto, di dare inizio a ricerche e studi per un edizione critica che garantisse l'autenticità e la fedeltà testuale delle Opere di Scoto. P. Efrem Longpré fu incaricato di presiedere e coordinare il primo gruppo di studiosi a Quaracchi.

${ }^{28}$ Cfr. Hechich, ,L'edizione critica delle opere”, 71.

${ }^{29}$ Il nome ufficiale della Commissione era in latino il seguente: „Commissio omnibus Operibus Ioannis Duns Scoti ad fidem codicum edendis”, accesso 24 gennaio 2021, https://www.scoto.net/ index.php/commissione/storia?start $=5$.

${ }^{30}$ Hechich, ,L'edizione critica delle opere”, 70.

${ }^{31}$ Commisione Scotista Internazionale, accesso 24 gennaio 2021, https://www.scoto.net/ index.php/commissione/pubblicazioni/7-elenco-opere-commissione. 
La Commissione Scotista Internazionale (così si chiama oggi) preparò una risposta „seria e documentata" ${ }^{32}$, raccolta in un volume di ben 365 pagine, che il 20 gennaio del 1970 consegnò alla Congregazione per le Cause dei Santi. La conferma dell'ortodossia degli scritti di Scoto, resa pubblica con decreto ufficiale il 4 maggio del 1972, ha permesso non solo la rivalutazione della solidità della dottrina di Duns Scoto, ma anche il proseguio della Causa per il riconoscimento del culto a lui tributato (il 6 luglio 1991) e quella di beatificazione con il tributo degli onori liturgici nella Basilica Vaticana nel 1993. Giovanni Paolo II infatti, il 20 marzo 1993, durante una pubblica e solenne celebrazione in San Pietro proclamò beato Giovanni Duns Scoto e stabilì l'8 novembre giorno della memoria liturgica ${ }^{33}$.

Il pontefice polacco già il 16 gennaio del 1982 aveva espresso parole di apprezzamento e sostegno per la scientificità degli scritti dello Scoto. Facendo allora visita alla Commissione Scotista, disse in quello occasione: „Finalmente posso metter piede in questo sacrario della scienza" ${ }^{4}$. I membri della Commissione raccontano che si soffermò anche allungo a parlare con loro, con interesse e passione per il lavoro da essi svolto ${ }^{35}$. Le parole però, che costituiscono un prezioso sigillo autografo di Papa Wojtyła a favore della grandezza intellettuale e la santità di Giovanni Duns Scoto, sono quelle che proferì il 16 febbraio del 2002 durante l'Udienza speciale concessa alla Commissione Scotista:

Duns Scoto, con la sua splendida dottrina sul primato di Cristo, sull'Immacolata Concezione, sul valore primario della Rivelazione e del Magistero della Chiesa, sull'autorità del Papa, sulla possibilità della ragione umana di rendere accessibili, almeno in parte le grandi verità della fede, di dimostrarne la non contradditorietà, rimane ancor oggi un pilastro della teologia cattolica, un Maestro originale e ricco di spunti e sollecitazioni per una conoscenza sempre più completa delle verità della fede ${ }^{36}$.

P. Orlando Todisco in uno dei suoi articoli, ripropone con accuratezza e enorme capacità di sintesi il pensiero di Giovanni Duns Scoto, partendo da una riflessione sulla ragione nella fede. A suo dire il Dottore Sottile fa parte

\footnotetext{
${ }^{32}$ Vedi Hechich, „L'edizione critica delle opere”, 71.

${ }^{33} \mathrm{Cfr}$. dati biografici confermati dalla Commissione Scotista Internazionale, accesso 24 gennaio 2021, https://www.scoto.net/index.php/duns-scoto/vita?start=4.

${ }^{34}$ Hechich, „L'edizione critica delle opere”, 65.

${ }^{35} \mathrm{Cfr}$. idem.

${ }^{36}$ „Acta Ordinis Fratrum Minorum” 121 (2002), 11.
} 
di quei teologi che scelgono di vivere una vita ai confini del tangibile e dell'empirico, muovendosi ai margini del linguaggio e del pensiero logico comune, rischiando di cadere nell'oscuro, nel paradossale, sovrarazionale e soprannaturale. Scoto si occupa di una teologia di carattere sistematico che vede Dio „al centro di” e ,in relazione con” ogni altro aspetto del discorso religioso, e che fa convergere il tutto verso di Lui, come termine-chiave dell'intero impianto teoretico-pratico ${ }^{37}$. Il pensiero del Sottile, dice, lo si può interpretare "come tentativo volto a recuperare quell'orizzonte teoreticoesistenziale del messaggio cristiano, che il rinato pensiero pagano, attraverso la teorizzazione di un progetto esistenziale, esclusivamente poco attento alla trascendenza, sembrava mettesse in pericolo"38. "Egli pertanto si muove in un ambito periferico rispetto alla definita chiarezza del discorso religioso pagano, premuroso di fare emergere quella tensione dialettica che respinge oltre l'area propriamente scientifica e filosofica, sostenuto dalla fede nella somma libertà di Dio. Dio non è abbassato al livello dell'uomo, entrambi chiusi in un orizzonte naturalistico, ma l'uomo elevato al livello di Dio"39. Scoto è convinto, in senso tipicamente agostiniano, che Dio, nonostante sia a noi intimo più di quanto lo siamo a noi stessi, tuttavia è percepibile soltanto nella misura in cui Egli voglia a noi rivelarsi (por. Mt 11, 27; Lc 10, 21-22). Egli non è oggetto naturale dell'intelletto, ma volontario, restando libero di manifestarsi come di nascondersi. Nell'ascesa a Dio dell'uomo, che sperimenta da una parte il suo limite, come anche il suo naturale tendere verso l'infinito, la filosofia non è in grado di aiutarci fino in fondo. Essa non è capace di introdurci nel sacrario di Dio, salvo che Lui stesso ci prenda per mano e ci conduca li. Da qui emerge per il Sottile la necessità e la centralità della Rivelazione ${ }^{40}$. Due infatti sono gli attributi che caratterizzano la prospettiva essenzialmente teologica del nostro Dottore: 1. il rifiuto del carattere totalitaristico della filosofia pagana; 2. Il bisogno della dottrina rivelata. Scoto vede questi due aspetti fusi in una paradossale unità, "folle", "incompossibile" e "sovrarazionale" 41 .

\footnotetext{
${ }^{37}$ Por. Todisco, „La ragione”, 261.

${ }^{38}$ Ibidem, 262.

${ }^{39}$ Ibidem.

${ }^{40} \mathrm{Cfr}$. ibidem.

${ }^{41}$ Vedi ibidem.
} 
Il Dio infinito dello Scoto, essenzialmente libero, imperscrutabile nell'essere e nell'operare, si trova lungo quella frontiera del linguaggio dove più che l'argomentazione conta l'allusione o meglio dove l'argomentazione è pervasa da forza allusiva, capace di farci cogliere i nostri limiti e di riflesso ciò che ci trascende $\mathrm{e}^{42}$.

Il discorso di Scoto è da lui seguito battuta dopo battuta "con l'ansia teologica" di difendere il messaggio rivelato dall'inganno presuntuoso di "insidie razionalistiche" che lasciano soltanto nell'uomo la sensazione falsa "dell'autosufficienza" "43. "Il linguaggio è spinto tanto lontano che più oltre sembra che cada nel puro non senso. Premendo contro i limiti dell'ordinario, egli ha tentato di dilatarne i confini attraverso il recupero di quel senso di gratuità e di libertà che caratterizza il rapporto di Dio agli uomini, e di quell'umiltà e impegno esistenziali, che sono il contrassegno della nostra risposta, sempre ambigua e perfettiva" ${ }^{44}$. In sintesi i termini della speculativa di Scoto, sono da una parte "Dio, suprema bontà e libertà", "che redime"; e dall'altra "l'uomo libero e redento" $[\ldots]$ "che cerca di stringerne la mano salvatrice" ${ }^{45}$.

\section{LA DOTTRINA}

La ricerca della verità da parte di Scoto è orientata dalla fede ed il suo pensare è un filosofare nella fede. Il motto anselmiano fides quaerens intellectum è sempre più attuale e valido per il Sottile.

La fede introduce nell'anima una particolare inquietudine di ricerca ai fini del chiarimento, ma mai conclusivo, dell'orizzonte teoretico e pratico [...]. Il carattere esistenziale, sempre presente, è reso però in un contesto di tale universalizzazione da sembrare del tutto assente. Il problema del male [...] è diluito nell'analisi dei vari stadi che la nostra natura ha attraversato (natura institutae, destitutae, restitutae). Visione questa che $[\ldots]$ contribuisce a disegnare un modello armonico di umanità prima intatto, poi decaduto e poi redento ${ }^{46}$.

\footnotetext{
${ }^{42}$ Ibidem, 262-3.

${ }^{43}$ Vedi ibidem, 263.

${ }^{44}$ Ibidem.

${ }^{45}$ Ibidem.

${ }^{46}$ Ibidem, 264.
} 
L'orientamento filosofico di Scoto ha un taglio prettamente teoretico, salvo il colorito storico dell'antropologia, gnoseologia, ontologia e teologia, procede lungo linee essenzialistiche. Egli ci tiene a usare nella disputa l'arma della distinzione unita ad argomenti razionali. Tuttavia nel confronto con lo stile molto esistenziale-confessionale di Agostino, non si può affermare rigidamente che il discorso di Scoto sia del tutto esente dall'esperienza esistenziale. A dimostrazione di ciò, gli esperti di Scoto invitano a rileggere gli scritti del Sottile facendo attenzione ai rilievi riguardanti la sua fisionomia interiore, la fedeltà alla Chiesa, il contributo al superamento dei momenti di tensione tra trono ed altare, potere temporale e spirituale ${ }^{47}$.

Si deve per onestà scientifica ammettere che la teologia di Giovanni Duns Scoto nei secoli passati è stata vittima di critiche, accuse poco fondate, pregiudizi ed stata spesso trincerata ai margini della teologia cattolica, prestandone troppo poca attenzione. Sin dal 1879 in particolare con l'Aeterni Patris di Leone XIII che sosteneva in maniera evidente la filosofia ed il pensiero di S. Tommaso d'Aquino, il tomismo diventa il codice ufficiale della teologia cattolica, strumento di coesione ecclesiastica e riferimento spesso citato dagli esponenti più autorevoli delle università cattoliche più emergenti. Ne menzioniamo solo alcune come l'Istituto cattolico di Lovanio (1894) con il suo padre fondatore, il pensatore belga (poi creato cardinale da Pio X), Désiré Mercier; 1'Università cattolica di Milano, fondata da padre Gemelli nel 1921, affiancata nella sua opera di propagazione del tomismo dalla rivista Neo-Scolastica; ricordiamo gli storici tomisti Francesco Olgiati e Amato Masnovo di Milano, i teorici come Umberto Antonio Padovani e Marino Gentile dell'Università di Padova; Jacques Maritain e Étienne Gilson in Francia ${ }^{48}$.

Todisco accenna ad una timida ripresa degli studi scotistici a partire dal 1923 con un lavoro di approfondimento della dottrina. Al 1927 risale invece la prima commissione critica scotista incentrata ad un lavoro critico sulle fonti ${ }^{49}$.

\footnotetext{
${ }^{47}$ Cfr. ibidem, 265.

${ }^{48}$ Cfr. Ibidem.

${ }^{49}$ Vedi Carlo Balić, „Note per la storia della sezione e poi commissione scotista per l'edizione critica delle opere di G. Duns Scoto", in Studies Honoring Ignatius Charles Brady, Friar Minor, a cura di Romano S. Almagno e Conrad L. Harkins (St. Bonaventure, N.Y.: The Franciscan Institute, 1976), 17-44. Come già detto in precedenza, all'inizio fu Padre Efrem Longpré a presiedere la commissione detta di Quaracchi. Padre Karl Balić lo sostituì invece nel 1938 e con quest'ultimo ebbe inizio la pubblicazione dell'edizione critica delle opere di Scoto.
} 


\section{IL PENSARE CREDENDO}

Il pensiero del Sottile ha dovuto subire accuse di tutti i tipi. Fu accusato di: panteismo, scetticismo, criticismo, arbitrarismo morale. I giudizi più duri furono fondamentalmente due, quello di: essere corrosivo e distruttivo di filosofie salde e pacifiche; e avversario sistematico del pensiero tradizionale tomista $^{50}$. Aumentano oggi gli studiosi convinti che bisogna cercare una risposta ai pregiudizi troppo avventati e poco fondati di molti, esaminando la fisionomia segreta dell'animo di Scoto, i documenti della Santa Sede, la cronaca storica della dedizione totale del Sottile al papa; se ad azzittire le accuse non siano ancora bastati né la fatica trentennale della Commissione né i volumi miscellanei ${ }^{51}$.

Giovanni Duns Scoto aprì con la sua teologia un nuovo varco di pensiero all'interno dell' autorevole tradizione tomisto-bonaventuriana. Nella storia di diffusione dello Spirito cristiano, in maniera propizia si inserisce la nascita nel 1265/1266 di Scoto, proprio quando Tommaso e Bonaventura si trovano all'apice della loro produzione scientifica. Scoto ebbe l'ardore ispirato di varcare nuovi orizzonti di pensiero, quando tutto sembrava fosse già stato detto, avendo alle spalle "oltre cinquanta dottori francescani, aristotelci, avicennisti e averroisti, platonici, agostiniani, moderati ed esagerati ed una lunga schiera di pensatori cattolici, arabi e giudei" ${ }^{52}$. Un altro figlio di San Francesco, a quarant'anni dalla Sua morte, sceglie la strada impegnativa di non accontentarsi di accodarsi al numero dei discepoli né di Tommaso né di Bonaventura, ma di riproporre sempre una sintesi dottrinale fuori del coro, che superi la contrapposizione tra agostiniani e aristotelici, di offrire una visione unitaria e comprensiva delle loro precedenti ispirazioni, rimanendo sempre fedele al messaggio cristiano ${ }^{53}$. Scoto è fondamentalmente un agostiniano con molti elementi aristotelici o il propagatore di un aristotelismo arricchito da alcune intuizioni agostiniane. È un "pensatore problematico, che agita e discute le questioni prima di risolverle o anzi per meglio risolverle" 54 . Egli non accetta a priori e superficialmente le proposte del passato, consapevole della fragilità del pensiero umano, sempre passibile di revisioni

\footnotetext{
${ }^{50}$ Vedi Emile C.A. Pluzanski, Saggio sulla filosofia del Duns Scoto, tr. di Augusto Alfani (Firenze: Tipografia Enrico Ariani, 1892).

${ }^{51}$ Cfr. Todisco, „La ragione”, 267-80.

${ }^{52}$ Cfr. ibidem, 267.

${ }^{53} \mathrm{Cfr}$. ibidem.

${ }^{54}$ Vedi ibidem.
} 
ed ampliamenti. Il suo pathos speculativo si pone l'obbiettivo "di spingere innanzi il pensiero ereditato, nutre il suo spirito critico, nella ricerca del vero" 55 . Sente il bisogno di cogliere la verità in forma più piena e da una prospettiva più comprensiva. Il suo metodo viene definito giustamente 'critico storico', poiché la sua dottrina si fa' ponte di dialogo con le voci più autorevoli del pensiero contemporaneo e aiuta a "discernere il caduco dall'immutabile, il certo dall'opinabile" ${ }^{56}$. "Ardito e semplice [...], egli cammina a testa alta fra i massimi geni dell'umanità. Umilmente attinge ai tesori della Città di Dio e del Monologo, delle Sentenze o delle Summe"57. Del resto critica ugualmente sia Aristotele che Platone, Avicenna e Averroè, come anche Tommaso e Bonaventura, Goffredo di Fontaines e Enrico di Gand „,in nome di un maggiore attivismo dell'intelletto e della volontà, alquanto estinto in alcuni, troppo esaltato in altri, e tutti in nome di una più radicale difesa della trascendenza di Dio e della libertà dell'uomo"58.

\section{SERVITORE DELLA VERITÀ}

Scoto è servitore della verità, cosa che lo obbliga a scegliere la prima a scapito della continuazione della tradizione. Riporta con onestà di indagine l'opinione altrui, presentandone un laborioso esame, da cui ricava conclusioni non del tutto definite, che lascia in sospeso. Lontano da un meschino partitismo, mostra il coraggio di distaccarsi anche dalla speculativa di Bonaventura "bandiera del suo Ordine". Il Dottore Sottile emerge per la rigorosità della ricerca. È mendicante dell'Infinito, che nei suoi scritti conserva le sembianze del Dio vivo della Scrittura. Le verità da lui proposte non sono sentenze astratte, ma il velo dietro cui si cela la "persona viva di Cristo, che viene proposto alla nostra contemplazione e al nostro amore" ${ }^{59}$. Lo spirito francescano di Scoto è incline al pensare pregando e al pregare pensando. Egli cerca con insistenza il giusto connubio tra l' intelletto e la fede. Al centro del programma e delle sue ricerche vi è il conoscere Dio. "La fede accompagna

\footnotetext{
${ }^{55}$ Ibidem.

${ }^{56}$ Ibidem

${ }^{57}$ Ibidem

${ }^{58}$ Ibidem.

${ }^{59}$ Vedi ibidem, 268.
} 
la ragione in tutta la sua parabola speculativa, con il compito di indicarle il Dio cui deve pervenire. La fede è orientativa, è l'assente sempre presente" ${ }^{\prime 60}$.

Egli ripensa il rapporto fede e ragione, ripercorre gli itinerari di ricerca percorsi dai filosofi pagani e da quelli cristiani, per ,,mettere in luce deviazioni, passaggi illogici o irrigidimenti speculativi che si fermano al di qua di Dio"61. È il caso di Aristotele. I suoi sostenitori infatti, ,non ne rispettano per intero la suprema perfezione e l'essenziale libertà creativa, come Enrico di Gand"62.

Duns Scoto è un vero teologo, perché è un uomo di Dio. Oltre alla mole delle opere scritte, si distingue per "la serietà e l'impegno di una vita consumata nella ricerca appassionata del vero" $"$.

Il Sottile presenta un'analisi contorta, caratterizzata dalla distinctio scotista complicata da molteplici quaestiones. Nel confronto con gli autorevoli predecessori che si distinguono per fluidità del dettato come Tommaso o per limpidezza di stile come Bonaventura, il suo pensiero è sinuoso, complesso, colmo di impennate improvvise, soste inattese, rapidi ritorni, a volte frondosi, eruditi. Lo stile è difficile, ma Scoto preferisce posporre alla chiarezza espositiva la riflessione. Egli sceglie volutamente uno stile scientificamente freddo, per poi far trasparire un grande ordine concettuale. La sua formazione sperimentalista e matematica di stampo oxfordiano ${ }^{64}$, evince di gran lunga e le parole sono aride, precise come numeri, non sono superflue o decorative ${ }^{65}$. "Duns Scoto è un genio nato, non per divulgare ma per approfondire il vero, desideroso di costruire un sistema più aperto o almeno più dinamico, nel colloquio rispettoso con quanti lo hanno preceduto nella nobile impresa" ${ }^{96}$.

\section{UOMO DI DIO}

Il discorso di Scoto si fa' esistenziale e psicologico, quando si occupa della tensione fra la l'ascesa a Dio e l'insoddisfazione intellettivo-volitiva dell'uomo, che anela al riposo spirituale definitivo. Questi sono tutti temi

\footnotetext{
${ }^{60}$ Ibidem, 268-9.

${ }^{61}$ Vedi ibidem, 269.

62 Ibidem.

${ }^{63}$ Ibidem.

${ }^{64}$ Todisco sostiene la tesi difesa da Brampton sulla formazione teologica di Scoto ad Oxford tra il 1288 e 1301. Vedi Brampton, Duns Scoto at Oxford, 1288-1301, 5.

${ }^{65}$ Cfr. Todisco, „La ragione”, 269.

${ }^{66}$ Ibidem.
} 
tipici della struttura scolastica tradizionale. Si evince lo sforzo degli uomini comuni nel passare dal finito verso l'infinito, e la tensione dell'anima umana, che spinta da esigenze interiori, cerca il suo pieno appagamento in Dio. È questa la serenità, di chi guarda il creato dalla dimensione del suo estremo traguardo ed asseconda il bisogno esistenziale di trascendersi nell'infinito di Dio, Esso è iscritto nel nostro spirito ${ }^{67}$. Scoto si inserisce nella suddetta questione tentando di occupare una posizione centrale ed al tempo stesso unitaria, rispetto alle due direzioni principali del pensiero classico: quella aposterioristica empirica, e quella aprioristica interiorista; l'aristotelicotomista e 1'agostiniano-bonaventuriana ${ }^{68}$. Per far questo, il Dottore si serve dell'argomento psicologico, riguardante la naturale proiezione dell'intelletto verso l'infinito. Egli intende modulare la sua tesi volontaristica, secondo la quale "intelletto e volontà, più che avvertire dissonanze si adagiano in quieto riposo nel sommo pensabile e nel Supremo Bene, senza sforzi, con diletto e prontezza (sine habitu, prompte et delectabiliter)" ${ }^{\prime 69}$. È questa la riformulazione della dialettica ascensionale. Essa è presentata con deciso accento volontaristico. Partendo dai beni caduchi si dirige verso il bene per essenza che è oggetto ultimo della nostra volontà. La tesi volontaristica sostiene la concreta ordinazione dell'essere umano al sommo bene e non sfocia affatto, come pensano alcuni, nell'irrazionale.

Nella dialettica ascensionale si rimarca il primato della volontà sulla ragione che non intralcia la rigorosità scientifica della dimostrazione, bensì ne svela il movente ultimo, sul piano della fondazione ontologica. È un voler anticipare nel discorso il traguardo, che solo sarà raggiunto al termine del procedimento, ponendolo come movente principale della ricerca. Questa viene da esso spronata e motivata positivamente ${ }^{70}$.

Scoto si impone di tentare l'unificazione delle due posizioni teoretiche medioevali, fondendo "psicologia e metafisica, pura teoresi e partecipazione esistenziale" in un composto unitario ${ }^{71}$.

L'ascesa a Dio è per il nostro Dottore un discorso unitario, in cui confluiscono esigenze aprioristiche e aposterioristiche.

\footnotetext{
${ }^{67}$ Cfr. ibidem, 270.

${ }^{68} \mathrm{Cfr}$. ibidem.

${ }^{69}$ Vedi ibidem,

${ }^{70} \mathrm{Cfr}$. ibidem, 270-1.

${ }^{71}$ Vedi idem, 271.
} 
La vita è contrassegnata da una permanente dualità o inadeguazione tra le attese e le conquiste, componibile solo sul piano di Dio trascendente. È come un dramma permanente che attesta che in tutto ciò siamo, vogliamo o facciamo, l'infinito è ovunque presente come un cuneo conficcato, non per dividere ma per rinserrare e consolidare ${ }^{72}$.

Si apprezzerebbe meglio l'intenzione conciliativa che pervade l'ascesa a Dio di Scoto, qualora ci si rammentasse della durezza e ostilità delle posizioni contrastanti fra gli argomenti degli aristotelico-tomisti, e quelli degli agostiniani, anselmiani, bonaventuriani.

„Egli infatti ha inteso fondere 1'apriorismo internistico di carattere platonico-bonaventuriano con l'aposteriorismo empirico di matrice aristotelico-tomista" ${ }^{73}$.

Il Sottile fa riferimento alle tensioni esistenzialistiche dell'esperienza umana e questo dopo aver concluso una serie di argomentazioni di tipo metafisico. Ciò denota, da una parte il rifiuto della tesi agostiniana aprioristica di porre Dio all'inizio del cammino dell'uomo, dall'altra invece, dimostra che Dio è comunque nascostamente presente nel procedere di tutti, "anche se solo in vetta ci si accorge che colui che è ultimo è anche il primo, colui che si cerca è colui che muove" ${ }^{\text {74 }}$.

\section{IN UBBIDIENZA AL PAPA ED ALLA CHIESA}

La Santa Sede ha attestato autorevolmente l'ortodossia dottrinale dello Scoto attraverso la Lettera apostolica Alma parens ${ }^{75}$, che Paolo VI indirizzo ai partecipanti del Congresso internazionale della filosofia scolastica, svoltosi ad Oxford ed Edimburgo dall'11 al 17 settembre 1966. Con la stessa la Chiesa rende omaggio "a un figlio che, in un periodo di gravi conflitti, dimostrò un fermo attaccamento al soglio pontificio" ${ }^{76}$. Il documento si pone in piena continuità con una "serie ininterrotta di decreti" pontifici, che nei secoli hanno approvato e raccomandato sia lo studio che l'insegnamento della dottrina scotista $^{77}$. Vale la pena menzionarli per rendersi conto dell'assonanza dello

\footnotetext{
72 Ibidem, 273.

${ }^{73}$ Ibidem.

${ }^{74}$ Vedi ibidem, 274.

${ }^{75}$ Vedi in merito un'analisi accurata: Kośla, Jan Duns Szkot, 22-35.

${ }^{76}$ Ibidem, 274.

${ }^{77}$ Ibidem.
} 
scotismo con il Magistero della Chiesa, a confronto con il parere contrario di chi ha avuto in merito opinioni discostanti e screditanti ${ }^{78}$.

Ecco l'elenco dei decreti pontifici dal XVI-XX sec.:

Alessandro VI, Breve Nupter attendentes (1501) - prescrive i quattro Libri delle Sentenze del Dottore Sottile o di altri insigni francescani, come testo ufficiale per formazione francescana nel triennio teologico, da adattare conformemente al grado e alle capacità intellettuali degli studenti;

S. Pio V, Costituzione Apostolica Illa nos (1568) - approvando le Costituzioni dei Francescani Conventuali, ordina di insegnare la teologia speculativa secondo la dottrina di Scoto;

Urbano VIII, Breve Alias (1634) - ordina di licenziare i lettori di filosofia e teologia che direttamente o indirettamente, con scritti o a voce, si discostano dal pensiero del Sottile;

Paolo V con un suo decreto dichiara immune da censura la dottrina scotista e comanda che nessuno osi probire arbitrariamente la pubblicazione dei suo testi autentici;

Benedetto XIII, Breve Ad pastorali dignitatis (1729) - elogia fra l'altro la somma teologica di Duns Scoto, redatta da Girolamo da Montefortino;

Benedetto XIV (1757) si compiace con il Generale Clemente da Palermo, che nel quadriennio teologico vengano insegnati i Trattati e le Questioni di Duns Scoto. Si narra che il Generale dell'Ordine abbia fornito al Pontefice un elenco accurato delle sue opere;

Pio VII, Breve Non raro in humanis (1823) confermando le Costituzioni urbaniste dei Minori Conventuali, prescrive ulteriormente di insegnare una teologia che rifletta lo spirito del pensiero di Duns Scoto;

Leone XIII, Bolla Felicitate quadam (1897), ordina di osservare "con scrupolosa fedeltà e costanza" le Costituzioni dei Frati Minori nelle quali di prescrive di insegnare la filosofia e la teologia attenendosi alla dottrina di Scoto e dell'antica scuola francescana;

Pio X (1908), complimentandosi con P. Garcia per la divulgazione del pensiero di Scoto e per l'opuscolo di meditazioni ispirato alla dottrina scotista, incoraggia anche gli altri francescani a combattere il modernismo ispirandosi alle intuizioni filosofiche e teologiche del Dottore Sottile.

${ }^{78}$ Todisco rinvia ad un esposizione più ampia del contenuto dei suddetti documenti in: Lexicon scholasticum philosophico-thelogicum, elaborato da M. F. Garcia sulla base delle opere e dei documenti su Duns Scoto, pubblicato nel 1910 Ad Claras Aquas (Quaracchi), 34-61. 
L'elenco numeroso dei consensi pontifici sulla dottrina scotista è una conferma esplicita della Chiesa nei confronti di Duns Scoto e della sua scuola. Le parole di Paolo VI dell'Alma parens ne ribadiscono ulteriormente l'autorevolezza e l'ortodossia dottrinale:

"Accanto alla cattedrale maestosa e principale di S. Tommaso d'Aquino, fra tre, c'è quella degna d'onore - sia pur dissimile per mole e struttura che elevò al cielo su ferme basi e con arditi pinnacoli l'ardente speculazione di G. Duns Scoto" "79.

Importante è ricordare gli appellativi con i quali gli studiosi di ogni tempo hanno omaggiato il Dottore Sottile: lima della verità; pietra che affina gli ingegni; fenice dei filosofi; miracolo di penetrazione; colonna della religione serafica; gloria della Sorbona ecc...

Secondo il parere del gesuita Bernard Jansen, raramente nel passato sia stato così deformata la figura di un personaggio come è invece avvenuto con lo Scoto. Sembra che egli sia stato "più ignorato che conosciuto" 80 . É. Gilson opponendosi decisamente ai critici della dottrina scotista dice che quanti hanno ridicolizzato Duns Scoto, lo hanno fatto per ignoranza e aggiunge che né 1 'hanno letto né capito ${ }^{81}$.

Bisogna ricordare due episodi poco conosciuti della vita di Duns Scoto, riguardanti le conseguenze subite a causa del suo deciso schierarsi a favore del papa, ai tempi del conflitto tra Bonifacio VIII e Filippo IV ${ }^{82}$ : 1. la firma insieme ad altri 86 frati di una petizione a sostegno del papa contro il re nel 1302 che causò il conferimento ritardato (1305) del titolo accademico di Maestro a conclusione del conflitto tra le parti interessate; 2. l'abbandono della cattedra di Parigi ed il trasferimento a Colonia nel 1305, a seguito della vittoria della disputa sull'Immacolata concezione di Maria (che inasprì il conflitto tra soglio pontificio ed autorità regia ai tempi del processo contro i Templari).

\footnotetext{
${ }^{79}$ Paolo VI, Lettera apostolica Alma parens (14.07.1966), Acta Apostolicae Sedis 58 (1966), 609-14.

${ }^{80}$ Vedi Todisco, „La ragione”, 276.

${ }^{81}$ Cfr. ibidem, 276. Cfr. Étienne Gilson, Giovanni Duns Scoto. Introduzione alle sue posizioni fondamentali (Milano: Edizioni Jaca Book, 2008), 2-4.

${ }^{82}$ Il conflitto fra papa Bonifacio VIII e Filippo IV è datato fra il 1296 ed il 1303. Ebbe inizio a causa della consacrazione episcopale per la diocesi di Pamiers, di una figura avversa al re Filippo a cui segui' uno scambio reciproco di accuse, fino alla congregazione di un'assemblea di dotti del clero aderenti alla politica del re contro il papa, che infangò l'autorità papale con calunnie di eresia, simmonia, immoralità, superstizione.
} 
Il Generale Gonsalvo è obbligato ad un certo punto a rimuovere il Maestro Scozzese dalla cattedra parigina per "sottrarlo alla bufera che stava per scatenarsi". Infatti la dottrina sull'Immacolata Concezione sembrò un pretesto sufficiente per accusare il Dottore Sottile di eresia da parte dei sostenitori del Re. Duns Scoto si trasferisce per ubbidienza a Colonia e "soffocò" 83 in questo modo ulteriori propositi di veto che non poté piu esternare. Giovanni Duns Scoto infatti moriva 1'8 novembre del 1308 moriva a Colonia, nel convento francescano, ove insegnava teologia. Le sue spoglie sono sepolte nell'annessa chiesa conventuale, la Minoritenkirche.

La prima attestazione della morte di Giovanni Duns Scoto proviene dalla fonte più diretta e autentica: il Necrologium dell'antico convento. Questo documento costituisce la prima attestazione della morte di Giovanni Duns Scoto. Essa è la fonte più diretta e autentica possibile. L'originale è andato perduto a causa delle spoliazioni e manomissioni del convento.

Siamo a conoscenza del testo, grazie alla sollecitudine di padre Matteo Ferchio, un francescano che nel gennaio del 1619, avendo tra le mani l'originale del necrologio, in occasione della traslazione delle ossa, ebbe la prontezza di riportare l'esatta dicitura latina. Egli la pubblico l'anno successivo nella sua Apologia Prima. Nel necrologio vi è scritto: "Rever. P.F. Ioannes Scotus Sacrae Theologiae Professor, Doctor Subtilis nominatus, quondam Lector Coloniae, qui obiit anno Domini 1308, sexto Idus Novembris" ${ }^{\prime 4}$.

\section{BIBLIOGRAFIA}

Alliney, Guido. Giovanni Duns Scoto. Introduzione al pensiero filosofico. Bari: Edizioni di Pagina, 2012.

Balić, Carlo. "Note per la storia della sezione e poi commissione scotista per l'edizione critica delle opere di G. Duns Scoto". In Studies Honoring Ignatius Charles Brady, Friar Minor, a cura di Romano S. Almagno e Conrad L. Harkins, 17-44. St. Bonaventure, N.Y.: The Franciscan Institute, 1976.

Boulnois, Olivier. Duns Scot, la rigueur de la charité. Paris: Les Éditions du Cerf, 1998.

Brampton, Charles K. "Duns Scotus at Oxford, 1288-1301". Franciscan Studies 24 (1964): 5-20.

Commissio Scotistica Internazionalis. Duns Scoto - dati biografici, opere, letteratura, le parole dei papi. Accesso 24 gennaio 2021. https://www.scoto.net/index.php/duns-scoto.

Commissio Scotistica Internazionalis. Storia della Commisione. Accesso 24 gennaio 2021. https:// www.scoto.net/index.php.

\footnotetext{
${ }^{83}$ Vedi Todisco, „La ragione”, 279.

${ }^{84}$ Accesso 24 gennaio 2021, https://www.scoto.net/index.php/duns-scoto/vita?start=4.
} 
Courtenay, William J. "Early Scotist at Paris”. Franciscan Studies 69 (2011): 175-229.

Courtenay, William J. „Scotus at Paris”. In Via Scoti. Methodologia ad mentem Joannis Duns Scoti. Atti del Congresso Scotistico Internazionale, Roma 9-11 marzo 1993, a cura di Leonardo Sileo, vol. 1, 149-63. Roma: Edizioni Antonianum, 1995.

Doctoris Subtilis et Mariani I. D. Scoti, Ordinis Fratrorum Minorum. Opera omnia. Studio et cura Commissionis Scotisticae ad fidem codicum edita, Ordinatio, t. 1-12. Romae: Civitas Vaticana, 1950-2010.

Fiorentino, Francesco. Il Prologo all'«Ordinatio» di Giovanni Duns Scoto. Introduzione, testo, traduzione e commento. Roma: Città nuova, 2016.

Ghisalberti, Alessandro. Giovanni Duns Scoto: filosofia e teologia. Milano: Biblioteca Francescana, 1995.

Gilson, Étienne. Giovanni Duns Scoto. Introduzione alle sue posizioni fondamentali. Milano: Edizioni Jaca Book, 2008.

Giovanni Paolo II. „Discorso - «In udienza dal Papa la Commissione Scotista»”. Acta Ordinis Fratrum Minorum 121 ( Ianuarii-Aprilis 2002): 10-11.

Hechich, Barnaba. „L'edizione critica delle opere del Beato Giovanni Duns Scoto. Traguardi e prospettive". In Blogostawiony Jan Duns Szkot 1308-2008. Materiaty Międzynarodowego Sympozjum Jubileuszowego z okazji 700-lecia śmierci bt. Jana Dunsa Szkota, Katolicki Uniwersytet Lubelski Jana Pawła II, 8-10 kwietnia 2008, a cura di Edward I. Zieliński OFMConv e Roman Maieran, 65-74. Lublin:Wydawnictwo KUL, 2010.

Kośla, Romuald H. Jan Duns Szkot. Jego dzieło i myśl od początku XX wieku do dzisiaj. Kraków: Wydawnictwo Unum, 2011.

Krąpiec, Mieczyslaw A. „Przesłanie”. In Btogosławiony Jan Duns Szkot 1308-2008. Materiały Międzynarodowego Sympozjum Jubileuszowego z okazji 700-lecia śmierci bt. Jana Dunsa Szkota, Katolicki Uniwersytet Lubelski Jana Pawła II, 8-10 kwietnia 2008, a cura di Edward I. Zieliński OFMConv e Roman Maieran, 19-21. Lublin: Wydawnictwo KUL, 2010.

Merino, José A. Storia della filosofia francescana. Milano: Edizioni Biblioteca Francescana, 1993.

Paolo VI. Lettera apostolica Alma parens (14 luglio 1966). Acta Apostolicae Sedis 58 (1966): 609-14.

Pluzanski, Emile C.A. Saggio sulla filosofia del Duns Scoto. Tradotto da Augusto Alfani. Firenze: Tipografia di Enrico Ariani, 1892.

Rigobello, Armando. Linee per una antropologia prescolastica. Padova: Antenore, 1972.

Scoto Giovanni Duns. „Collectationes Oxonienses et Parisienses”. In Opera omnia, a cura di Giovanni Lauriola, 3 voll. Bari: AGA Alberobello, 1996-2001, II/1 (1998).

Steenberghen, Fernand van. „La philosophie à la veille de l'entrée en scène de Jean Duns Scot”. In Atti del Congresso scotistico internazionale. De doctrine J. Duns Scoti, a cura di Leonardo Sileo, vol. 1, 65-74. Roma: Edizioni Antonianum, 1968.

Todisco, Orlando. „La ragione nella fede secondo G. Duns Scoto”. Miscellanea Francescana 77 (1977): 261-96.

Vos, Antonie. The philosophy of John Duns Scotus. Edinburgh: Edinburgh University Press, 2006.

Wilk, Stanisław. „Siedemsetlecie śmierci bł. Jana Dunsa Szkota”. In Błogosławiony Jan Duns Szkot 1308-2008. Materiaty Międzynarodowego Sympozjum Jubileuszowego z okazji 700-lecia śmierci bł. Jana Dunsa Szkota, Katolicki Uniwersytet Lubelski Jana Pawła II, 8-10 kwietnia 2008, a cura di Edward I. Zieliński OFMConv e Roman Maieran, 13-5. Lublin: Wydawnictwo KUL, 2010. 
Wolter, Allan B. „Duns Scotus at Oxford”. In Via Scoti. Methodologia ad mentem Joannis Duns Scoti. Atti del Congresso Scotistico Internazionale, Roma 9-11 marzo 1993, a cura di Leonardo Sileo, vol. 1, 183-92. Roma: PAA-Edizioni Antonianum, 1995.

Zavalloni, Roberto. Giovanni Duns Scoto. Maestro di vita e pensiero. Bologna: Bologna Edizioni Francescane, 1992.

\title{
GIOVANNI DUNS SCOTO: \\ UN TEOLOGO INCOMPRESO, UN SANTO DA RISCOPRIRE
}

\author{
Som mario
}

Nell'approccio al pensiero di Giovanni Duns Scoto, ci si imbatte in maniera inevitabile con la luminosa testimonianza di vita di un grande santo. Si ha a che fare con "un insieme inseparabile di filosofia, teologia e spiritualità". Giovanni Duns Scoto, filosofo e teologo del XIII secolo è un autore controverso e originale. Le sue opere in passato hanno avuto più critici e oppositori che sostenitori e discepoli. È un pensatore più moderno di quanto ci si possa immaginare. Non è per niente uno fra i tanti teologi e filosofi del passato, né i suoi scritti servono solo ad impolverare le nostre biblioteche. „Non è un fossile da ammirare, ma è un Maestro vivo e palpitante, pieno di ardore e di preziose intuizioni" di grande attualità. Questo francescano di origine scozzese, nato tra il 1265/66 e morto a Colonia l's novembre del 1308 lontano dalla sua patria, è un teologo ed un santo di grande spessore che solo oggi forse possiamo iniziare ad apprezzare.

Parole chiave: teologia; santità; dottrina; ragione; fede.

\section{JAN DUNS SZKOT: \\ NIEZROZUMIANY TEOLOG, WCIĄŻ NIEODKRYTY ŚWIĘTY}

$$
\text { Streszczenie }
$$

Badając myśl Jana Dunsa Szkota, nieuchronnie napotyka się na świetlane świadectwo życia tego wielkiego świętego. Mamy tu do czynienia z „nierozerwalną całością filozofii, teologii i duchowości”. Jan Duns Szkot, filozof i teolog żyjący w XIII wieku, jest autorem kontrowersyjnym i oryginalnym. Jego twórczość w przeszłości miała więcej krytyków i przeciwników niż zwolenników i uczniów. Nie jest on bynajmniej jednym z wielu teologów i filozofów przeszłości, a jego pisma nie służą jedynie do zbierania kurzu w naszych bibliotekach. „Nie jest on skamieliną, którą można podziwiać, ale jest żywym i zachwycającym Mistrzem, pełnym zapału i cennych spostrzeżeń" o wielkiej aktualności. Jego myśl jest bardziej nowoczesna, niż można by przypuszczać. Ten franciszkanin szkockiego pochodzenia, który urodził się w 1265 lub 1266 r., a zmarł 8 listopada 1308 r. w Kolonii, daleko od swojej ojczyzny, jest teologiem i świętym o wielkiej głębi, którą być może dopiero dzisiaj zaczynamy dostrzegać.

Słowa kluczowe: teologia; świętość; doktryna; rozum; wiara. 